\title{
ON A PROBLEM OF BERNIK, KLEINBOCK AND MARGULIS
}

\author{
NATALIA BUDARINA* \\ Department of Mathematics and Statistics, NUI Maynooth, Maynooth, Co. Kildare, Ireland \\ e-mail: buda77@mail.ru
}

(Received 29 March 2010; revised 9 September 2010; accepted 11 April 2011)

\begin{abstract}
In this paper, the Khintchine-type theorems of Beresnevich (Acta Arith. 90 (1999), 97) and Bernik (Acta Arith. 53 (1989), 17) for polynomials are generalised to incorporate a natural restriction on derivatives. This represents the first attempt to solve a problem posed by Bernik, Kleinbock and Margulis (Int. Math. Res. Notices 2001(9) (2001), 453). More specifically, the main result provides a probabilistic criterion for the solvability of the system of inequalities $|P(x)|<\Psi_{1}(H)$ and $\left|P^{\prime}(x)\right|<\Psi_{2}(H)$ in integral polynomials $P$ of degree $\leq n$ and height $H$, where $\Psi_{1}$ and $\Psi_{2}$ are fairly general error functions. The proof builds upon Sprindzuk's method of essential and inessential domains and the recent ideas of Beresnevich, Bernik and Götze (Compositio Math. 146 (2010), 1165) concerning the distribution of algebraic numbers.
\end{abstract}

2010 Mathematics Subject Classification. 11J83, 11K60, 11J13

1. Introduction. First some notation. Throughout this paper, $\Psi_{i}: \mathbb{R}^{+} \rightarrow \mathbb{R}^{+}(i=$ $1,2)$ denote real positive functions, $\# S$ stands for the cardinality of $S$ and $\mu$ denotes Lebesgue measure in $\mathbb{R}$. Given an interval $J \subset \mathbb{R},|J|$ will denote the length of $J$. Also, $B(x, \rho)$ will denote the interval in $\mathbb{R}$ centred at $x$ of radius $\rho$. Given $n \in \mathbb{N}, P_{n}$ will be the set of integral polynomials of degree $\leq n$. Given a polynomial $P, H(P)$ will denote the maximum of the absolute values of its coefficients and referred to as the height of $P$. By $\ll(\gg)$ we will mean the Vinogradov symbol with the implicit constant depending on $n$ only. We shall write $a \asymp b$ when the inequalities $a \ll b$ and $a \gg b$ hold simultaneously.

In this paper, we will be concerned with the measure theoretic properties of the set

$$
\mathcal{P}_{n}\left(\Psi_{1}, \Psi_{2}\right)=\left\{x \in\left[-\frac{1}{2}, \frac{1}{2}\right]: \begin{array}{l}
|P(x)|<\Psi_{1}(H(P)) \\
\left|P^{\prime}(x)\right|<\Psi_{2}(H(P))
\end{array} \text { for i.m. } P \in P_{n}\right\},
$$

where 'i.m.' stands for 'infinitely many'.

Using the fact that $|x| \leq 1 / 2$, one easily verifies that the bottom line inequality in (1) imposes no restriction when $\Psi_{2}(H) \geq 4 H$. In this case, the set $\mathcal{P}_{n}\left(\Psi_{1}, \Psi_{2}\right)$ is characterised by the first inequality only. Hence, for simplicity, $\mathcal{P}_{n}\left(\Psi_{1}, 4 H\right)$ will be denoted by $\mathcal{P}_{n}\left(\Psi_{1}\right)$. Thus,

$$
\mathcal{P}_{n}\left(\Psi_{1}\right)=\left\{x \in\left[-\frac{1}{2}, \frac{1}{2}\right]:|P(x)|<\Psi_{1}(H(P)) \text { for i.m. } P \in P_{n}\right\} .
$$

The measure theoretic characterisation of the set $\mathcal{P}_{n}\left(\Psi_{1}\right)$ has been the catalysis of the development of metric number theory over the last century. In the case $n=1$, it was

*Supported by the Science Foundation Ireland grant SFI-RFP08/MTH1512. 
first described in 1924 by Khintchine [23] in his beautiful discovery of a zero-one law stated below in a slightly stronger modern version, see [8].

THEOREM (Khintchine [23]).

$$
\mu\left(\mathcal{P}_{1}(\Psi)\right)=\left\{\begin{array}{lll}
0 & \text { if } & \sum_{h=1}^{\infty} \Psi(h)<\infty \\
1 & \text { if } & \sum_{h=1}^{\infty} \Psi(h)=\infty \text { and } \Psi(h) \text { is monotonic. }
\end{array}\right.
$$

When $n>1$, the measure theoretic characterisation of $\mathcal{P}_{n}(\Psi)$ goes back to the conjecture of Mahler (1932) that $\mathcal{P}_{n}(\Psi)$ has zero measure whenever $\Psi(h)=h^{-n-\varepsilon}$ for any $\varepsilon>0$. The conjecture was studied in depth for over 30 years and proved in full generality by Sprindzuk in 1965, see [25]. The problem of establishing an analogue of Khintchine's theorem, this time for polynomial approximations, is attributed to Alan Baker [2]. The complete solution to Baker's problem was given by Bernik [13] (convergence case) and Beresnevich [3] (divergence case). These results assume that $\Psi$ is monotonic. However, more recently, Beresnevich [5] has shown that the condition that $\Psi$ is monotonic can be dropped from the convergence case, thus establishing the following complete analogue of Khintchine's theorem for all degrees $n$ :

THEOREM (Beresnevich $[3,5])$. For any $n \in \mathbb{N}$

$$
\mu\left(\mathcal{P}_{n}(\Psi)\right)=\left\{\begin{array}{lll}
0 & \text { if } & \sum_{h=1}^{\infty} h^{n-1} \Psi(h)<\infty, \\
1 & \text { if } \quad \sum_{h=1}^{\infty} h^{n-1} \Psi(h)=\infty \text { and } \Psi(h) \text { is monotonic. }
\end{array}\right.
$$

Bearing in mind the monotonicity of $\Psi$, Khintchine-type theorems for polynomials have been subsequently established in the case of complex [20] and $p$-adic [12] variables and for simultaneous approximation in $\mathbb{R}, \mathbb{C}$ and $\mathbb{Q}_{p}[\mathbf{1 5}, \mathbf{1 6}, \mathbf{1 7}]$. Also, in the case of monic polynomials, Khintchine-type results have been obtained in [19] and [22]. In the more general case of Diophantine approximation on non-degenerate manifolds, Khintchine-type theorems have been established in $[4, \mathbf{1 1}, \mathbf{1 8}]$ and for the inhomogeneous case in [1]. Furthermore, it has been shown in [21] that the monotonicity of $\Psi$ can be omitted in the case of Diophantine approximation on arbitrary non-degenerate curves. Also, recently Khintchine-type theorems have been established in the case of simultaneous Diophantine approximation, see $[\mathbf{6 , 9}, \mathbf{9}, \mathbf{2 6}]$ and references therein.

Understanding the behaviour of derivatives of polynomials (and more generally linear forms of smooth functions) has been somewhat crucial within the above advances. Building upon the landmark results [24] of Kleinbock and Margulis, in 2001, Bernik et al. [18] found a far-reaching generalisation of Sprindzuk's theorem (Mahler's conjecture), which incorporated a condition on derivatives of this ilk. Their theorem treats linear forms of non-degenerate families of functions and in the case of polynomials reduces to the following statement.

TheOrem (Bernik, Kleinbock \& Margulis [18]). For any $n \in \mathbb{N}$

$$
\mu\left(\mathcal{P}_{n}\left(\Psi_{1}, \Psi_{2}\right)\right)=0
$$

when $\Psi_{1}(h)=h^{-w-\lambda}$ and $\Psi_{2}(h)=h^{1-\lambda}$ for some $\lambda \geq 0$ and $w>n-2 \lambda$. 
In the same paper, Bernik et al. set up a problem about finding optimal conditions on $\Psi_{1}$ and $\Psi_{2}$ which ensure the validity of (4). In other words, they asked for establishing a Khintchine-type theorem for $\mathcal{P}_{n}\left(\Psi_{1}, \Psi_{2}\right)$. The present paper represents the first attempt to tackle this obviously hard and demanding problem.

Before we state the main result, it is convenient to introduce some auxiliary functions, which are determined by $\Psi_{1}$ and $\Psi_{2}$ and carry various 'technical' restrictions on $\Psi_{1}$ and $\Psi_{2}$. At this point, it is enough to say that these auxiliary functions naturally arrear in the proof. They are

$$
\begin{aligned}
\bar{\Psi}_{1}(h) & =\min \left\{\Psi_{1}(h), \Psi_{2}^{-1}(h) h^{1-n}\right\} \\
\psi(h) & =K^{-1} \bar{\Psi}_{1}(h) \Psi_{2}^{-1}(h), \\
\rho(h) & =K^{2} h^{-n+1} \Psi_{2}^{-2}(h)
\end{aligned}
$$

where $K=\left(3 v^{-1}\right)^{n-1} c_{1} n$ is a sufficiently large constant depending on $n$ only.

Following Beresnevich, Dickinson and Velani [8], we will say that a function $f$ is 2-regular if there is a positive $\lambda<1$ such that $f\left(2^{t+1}\right) \leq \lambda f\left(2^{t}\right)$ for all sufficiently large $t$. Also, we will say that $f$ is quasi-monotonic if there are constants $z$ and $c_{1}$ such that $0<z<1 \leq c_{1}$ and $f(z x) \leq c_{1} f(x)$ for all sufficiently large $x$.

THEOREM 1. Let $\Psi_{1}, \Psi_{2}: \mathbb{R}^{+} \rightarrow \mathbb{R}^{+}$be such that $\Psi_{1} \Psi_{2}$ is a monotonically decreasing function. Let $n \geq 2$ be an integer.

(1) Suppose that $\Psi_{2}(h) \geq h^{1 / 2+\epsilon}, \epsilon>0$, then

$$
\mu\left(\mathcal{P}_{n}\left(\Psi_{1}, \Psi_{2}\right)\right)=0 \quad \text { if } \quad \sum_{h=1}^{\infty} h^{n-2} \Psi_{1}(h) \Psi_{2}(h)<\infty
$$

(2) Suppose that $K h^{\frac{-n+2}{3}} \leq \Psi_{2}(h)<k_{0} K h$ and that $\psi$ or $\rho$ is 2-regular, where $k_{0}$ is a positive constant depending on $n$ only. Furthermore, suppose that $\psi$ is quasimonotonic. Then

$$
\mu\left(\mathcal{P}_{n}\left(\Psi_{1}, \Psi_{2}\right)\right)=1 \quad \text { if } \quad \sum_{h=1}^{\infty} h^{n-2} \Psi_{1}(h) \Psi_{2}(h)=\infty
$$

2. Proof of Theorem 1: the divergence case. The proof of Case (2) of Theorem 1 is based on two techniques: the ubiquity technique of Beresnevich et al. [8] and the construction of Beresnevich, Bernik and Götze [10] of a system of close conjugate algebraic numbers. The construction of Beresnevich et al. [10] is modified to suite our main goal. Throughout this paper, we deal with algebraic numbers in $\mathbb{C}$. Let $n \geq 2$. Recall that complex algebraic numbers are called conjugate (over $\mathbb{Q}$ ) if they are roots of the same irreducible (over $\mathbb{Q}$ ) polynomial with rational integer coefficients. Here and elsewhere, $H(\alpha)$ denotes the height of an algebraic number $\alpha$ defined to be the absolute height of the minimal polynomial of $\alpha$ over $\mathbb{Z}$. 
We begin by stating the following important auxiliary result established as Lemma 4 in [10]. In what follows, $\xi_{0}, \ldots, \xi_{n} \in \mathbb{R}^{+}$will satisfy the conditions

$$
\begin{aligned}
& \xi_{i} \ll 1, \quad \text { when } 0 \leq i \leq m-1, \\
& \xi_{i} \gg 1, \quad \text { when } m \leq i \leq n, \\
& \xi_{0}<\varepsilon, \quad \xi_{n}>\varepsilon^{-1},
\end{aligned}
$$

for some $0<m \leq n$ and $\varepsilon>0$, where the implied constants depend on $n$ only. Assume also that

$$
\prod_{i=0}^{n} \xi_{i}=1
$$

LEMMA 1. For every $n \geq 2$, there are positive constants $\delta_{0}$ and $c_{0}$ depending on $n$ only with the following property. For any interval $J \subset\left[-\frac{1}{2}, \frac{1}{2}\right]$ there is a sufficiently small $\varepsilon=\varepsilon(n, J)>0$ such that for any $\xi_{0}, \ldots, \xi_{n}$ satisfying (5) and (6) there is a measurable set $G_{J} \subset J$ satisfying

$$
\mu\left(G_{J}\right) \geq \frac{3}{4}|J|
$$

such that for every $x \in G_{J}$ there are $n+1$ linearly independent primitive irreducible polynomials $P \in \mathbb{Z}[x]$ of degree exactly $n$ such that

$$
\delta_{0} \xi_{i} \leq\left|P^{(i)}(x)\right| \leq c_{0} \xi_{i} \quad \text { for all } \quad i=0, \ldots, n .
$$

Furthermore, let $0<v<1, Q>1$ and $\mathcal{A}_{n, v}(Q)$ be the set of algebraic numbers $\alpha_{1} \in \mathbb{R}$ of degree $n$ and height $H\left(\alpha_{1}\right)$ satisfying

$$
\nu Q \leq H\left(\alpha_{1}\right) \leq v^{-1} Q
$$

such that

$$
v \leq \frac{\left|\alpha_{1}-\alpha_{2}\right|}{Q^{-1} \Psi_{2}(Q)} \leq v^{-1} \quad \text { for some } \alpha_{2} \in \mathbb{R} \text { conjugate to } \alpha_{1} .
$$

The following lemma generalises [10, Theorem 2] and represents the stepping stone for establishing the divergence case of Theorem 1.

LEMMA 2. Let $n \geq 2$ be an integer and let $\Psi_{2}: \mathbb{R}^{+} \rightarrow \mathbb{R}^{+}$satisfy the inequality $Q^{\frac{-n+2}{3}} \leq \Psi_{2}(Q)<k_{0} Q$ for all $Q \in \mathbb{N}$ and some constant $k_{0}>0$. Then, there is a constant $v>0$ depending on $n$ only such that for any interval $J \subset\left[-\frac{1}{2}, \frac{1}{2}\right]$, for all sufficiently large $Q$

$$
\mu\left(\bigcup_{\alpha_{1} \in \mathcal{A}_{n, v}(Q)} B\left(\alpha_{1}, Q^{-n+1} / \Psi_{2}^{2}(Q)\right) \cap J\right) \geq \frac{3}{4}|J|
$$


Proof. The proof will follow the line of arguments of [10, Theorem 2]. Let $\delta_{0}$ and $c_{0}$ be the same as in Lemma 1. Define the following parameters:

$$
\xi_{0}=\eta Q^{-n+1} \Psi_{2}^{-1}(Q), \quad \xi_{1}=\eta^{-n} \Psi_{2}(Q), \quad \xi_{i}=\eta Q \quad(2 \leq i \leq n),
$$

where $0<\eta<1$ is a sufficiently small fixed parameter depending on $n$ only which will be specified later. Fix any interval $J \subset\left[-\frac{1}{2}, \frac{1}{2}\right]$ and let $\varepsilon=\varepsilon(n, J)$ be the same as in Lemma 1 . Then, (5) is satisfied with $m \in\{1,2\}$ for sufficiently large $Q$. Also, the validity of (6) easily follows from (12). Let $G_{J}$ be the set arising from Lemma 1 and $x \in G_{J}$. Then, by Lemma 1 , there is a primitive irreducible polynomial $P \in \mathbb{Z}[x]$ of degree $n$ satisfying (8).

Finding $\alpha_{1}$. Let $y \in \mathbb{R}$ be such that $|y-x|=Q^{-n+1} \Psi_{2}^{-2}(Q)$. Using the fact that $\Psi_{2}(Q) \geq$ $Q^{\frac{-n+2}{3}}$, we have $|y-x|<1$. Furthermore, by Taylor's formula,

$$
P(y)=\sum_{i=0}^{n} \frac{1}{i !} P^{(i)}(x)(y-x)^{i}
$$

Using the inequality $|x-y|<1, \Psi_{2}(Q) \geq Q^{\frac{-n+2}{3}}$, (8) and (12), we verify that

$$
\left|P^{(i)}(x)(y-x)^{i}\right| \leq \eta c_{0} Q^{-n+1} \Psi_{2}^{-1}(Q) \quad \text { for } \quad i \geq 2 .
$$

Also, by (8) and (12), $|P(x)| \leq \eta c_{0} Q^{-n+1} \Psi_{2}^{-1}(Q)$. Therefore,

$$
\sum_{i \neq 1}\left|\frac{1}{i !} P^{(i)}(x)(y-x)^{i}\right| \leq \eta c_{0} Q^{-n+1} \Psi_{2}^{-1}(Q) \sum_{i=0}^{n} \frac{1}{i !}<3 \eta c_{0} Q^{-n+1} \Psi_{2}^{-1}(Q) .
$$

On the other hand,

$$
\left|P^{\prime}(x)(y-x)\right| \stackrel{(8) \&(12)}{\geq} \delta_{0} \eta^{-n} \Psi_{2}(Q) Q^{-n+1} \Psi_{2}^{-2}(Q) \geq \delta_{0} \eta^{-1} Q^{-n+1} \Psi_{2}^{-1}(Q) .
$$

It follows from (15) and (16) that $P(y)$ has different signs at the endpoints of the interval $|y-x| \leq Q^{-n+1} \Psi_{2}^{-2}(Q)$ provided that $\eta \leq \frac{1}{2} \delta_{0}^{1 / 2} c_{0}^{-1 / 2}$. By the continuity of $P$, there is a root $\alpha_{1}$ of $P$ in this interval, that is

$$
\left|x-\alpha_{1}\right|<Q^{-n+1} \Psi_{2}^{-2}(Q) .
$$

Finding $\alpha_{2}$. Let $y_{\rho}=x+\rho Q^{-1} \Psi_{2}(Q)$, where $2 \leq|\rho|<Q^{1 / 2} \Psi_{2}^{-1 / 2}(Q)$. For $\rho$ to exist one should impose the condition

$$
\Psi_{2}(Q)<Q / 4
$$

Now we will again use (13), this time with $y=y_{\rho}$. Using $|x-y|<1,|\rho|<$ $Q^{1 / 2} \Psi_{2}^{-1 / 2}(Q),(8)$ and (12), we verify that

$$
\left|P^{(i)}(x)\left(y_{\rho}-x\right)^{i}\right|<\eta|\rho| c_{0} Q^{-1} \Psi_{2}^{2}(Q) \quad \text { for } \quad i \geq 3 .
$$

By (8), (12) and the facts that $\Psi_{2}(Q) \geq Q^{\frac{-n+2}{3}}$ and $|\rho| \geq 2$, we have that

$$
|P(x)| \leq \eta c_{0} Q^{-n+1} \Psi_{2}^{-1}(Q) \leq|\rho| \eta c_{0} Q^{-1} \Psi_{2}^{2}(Q)
$$


and

$$
\left|P^{\prime}(x)\left(y_{\rho}-x\right)\right| \leq \eta^{-n} c_{0} \Psi_{2}(Q)|\rho| Q^{-1} \Psi_{2}(Q)=\eta^{-n} c_{0}|\rho| Q^{-1} \Psi_{2}^{2}(Q) .
$$

The latter two estimates together with (19) give

$$
\sum_{i \neq 2}\left|\frac{1}{i !} P^{(i)}(x)\left(y_{\rho}-x\right)^{i}\right| \leq \eta^{-n}|\rho| c_{0} Q^{-1} \Psi_{2}^{2}(Q) \sum_{i=0}^{n} \frac{1}{i !}<3 \eta^{-n}|\rho| c_{0} Q^{-1} \Psi_{2}^{2}(Q) .
$$

On the other hand,

$$
\left|\frac{1}{2 !} P^{\prime \prime}(x)\left(y_{\rho}-x\right)^{2}\right| \stackrel{(8) \&(12)}{\geq} \frac{1}{2} \delta_{0} \eta Q|\rho|^{2} Q^{-2} \Psi_{2}^{2}(Q)=\frac{1}{2} \delta_{0} \eta \rho^{2} Q^{-1} \Psi_{2}^{2}(Q) .
$$

It follows from (20) and (21) that $P(y)$ has the same signs at the points $y_{ \pm \rho_{0}}$ (same as $\left.P^{\prime \prime}(x)\right)$ with $\rho_{0}=7 c_{0} \eta^{-n-1} \delta_{0}^{-1}$. Obviously, $\rho_{0}$ should satisfy the inequality $\rho_{0}<$ $Q^{1 / 2} \Psi_{2}^{-1 / 2}(Q)$. This imposes another condition on $\Psi_{2}$. Together with the estimation (18) this gives

$$
\Psi_{2}(Q)<k_{0} Q \quad \text { with } k_{0}=\min \left\{\frac{1}{4}, \frac{1}{\rho_{0}^{2}}\right\} .
$$

On the other hand, arguing the same way as during 'Finding $\alpha_{1}$ ', one readily verifies that $P\left(y_{2}\right)$ and $P\left(y_{-2}\right)$ have different signs. Therefore, $P(y)$ changes sign on one of the intervals

$$
\left[-\rho_{0} Q^{-1} \Psi_{2}(Q),-2 Q^{-1} \Psi_{2}(Q)\right] \quad \text { or } \quad\left[2 Q^{-1} \Psi_{2}(Q), \rho_{0} Q^{-1} \Psi_{2}(Q)\right] .
$$

By the continuity of $P$, there is a root $\alpha_{2}$ of $P$ in that interval, that is

$$
2 Q^{-1} \Psi_{2}(Q) \leq\left|x-\alpha_{2}\right|<\rho_{0} Q^{-1} \Psi_{2}(Q) .
$$

Combining (17) and (22) gives $Q^{-1} \Psi_{2}(Q) \leq\left|\alpha_{1}-\alpha_{2}\right| \leq\left(\rho_{0}+1\right) Q^{-1} \Psi_{2}(Q)$, thus establishing (10).

Estimates for the height. Using the fact that $|x| \leq \frac{1}{2}$, (8) and (12) we verify that

$$
\begin{aligned}
& \left|a_{n}\right|=\left|\frac{1}{n !} P^{(n)}(x)\right| \asymp Q, \\
& \left|a_{n-1}\right|=\left|\frac{1}{(n-1) !} P^{(n-1)}(x)-n a_{n} x\right| \ll Q, \\
& \left|a_{k}\right|=\left|\frac{1}{k !} P^{(k)}(x)-\sum_{i=k+1}^{n} \frac{i !}{k !(i-k) !} a_{i} x^{i-k}\right| \ll Q, \text { for } 0 \leq k \leq n-2 .
\end{aligned}
$$

The upshot is that $H\left(\alpha_{1}\right) \asymp Q$. This establishes (9) and completes the proof of Lemma 2.

REMARK 1 . From the fact that $\left|a_{n}\right| \asymp Q$ and from the well-known property that $\left|\alpha_{i}\right| \ll H\left(\alpha_{i}\right) /\left|a_{n}\right|$ (see [25]), it follows that any $\alpha_{i}$ conjugate to $\alpha_{1}$ is bounded by a constant depending on $n$ only. 
We will use the ubiquitous systems technique, which is now briefly recalled in a simplified form (see [8] for more details and [7] for the related notion of regular systems). Let $I$ be an interval in $\mathbb{R}$ and $\mathcal{R}:=\left(r_{\alpha}\right)_{\alpha \in J}$ be a family of points $r_{\alpha}$ in $I$ indexed by a countable set $\mathcal{J}$. Let $\beta: \mathcal{J} \rightarrow \mathbb{R}^{+}: \alpha \mapsto \beta_{\alpha}$ be a function on $\mathcal{J}$, which attaches a 'weight' $\beta_{\alpha}$ to points $r_{\alpha}$. For $t \in \mathbb{N}$, let $\mathcal{J}(t):=\left\{\alpha \in \mathcal{J}: \beta_{\alpha} \leq 2^{t}\right\}$ and assume $\mathcal{J}(t)$ is always finite.

Let $\rho: \mathbb{R}^{+} \rightarrow \mathbb{R}^{+}$be a function such that $\lim _{t \rightarrow \infty} \rho(t)=0$ referred to as ubiquity function. The system $(\mathcal{R} ; \beta)$ is called locally ubiquitous in I relative to $\rho$ if there is an absolute constant $m_{0}>0$ such that for any interval $J \subset I$

$$
\liminf _{t \rightarrow \infty} \mu\left(\bigcup_{\alpha \in \mathcal{J}(t)} B\left(r_{\alpha}, \rho\left(2^{t}\right)\right) \cap J\right) \geq m_{0}|J| .
$$

Given a function $\psi: \mathbb{R}^{+} \rightarrow \mathbb{R}^{+}$, let

$$
\Lambda_{\mathcal{R}}(\psi):=\left\{x \in I:\left|x-r_{\alpha}\right|<\psi\left(\beta_{\alpha}\right) \text { holds for infinitely many } \alpha \in \mathcal{J}\right\} .
$$

The following lemma is a modification of Theorem 1 in [8].

Lemma 3. Let $(\mathcal{R}, \beta)$ be a locally ubiquitous system in $J_{0}$ relative to $\rho$. Let the function $\psi$ or $\rho$ is 2-regular. Then $\mu\left(\Lambda_{\mathcal{R}}(\psi)\right)=\left|J_{0}\right|$ if $\sum_{t=1}^{\infty} \frac{\psi\left(2^{t}\right)}{\rho\left(2^{t}\right)}=\infty$.

Consider the function $\Psi_{3}(h)=\bar{\Psi}_{1}(h) \Psi_{2}(h)$, which is non-increasing. The monotonicity of $\Psi_{3}$ easily follows from the inequality $\min \left\{\Psi_{1}\left(h_{1}\right) \Psi_{2}\left(h_{1}\right) ; h_{1}^{1-n}\right\} \geq$ $\min \left\{\Psi_{1}\left(h_{2}\right) \Psi_{2}\left(h_{2}\right) ; h_{2}^{1-n}\right\}$ for all $h_{2} \geq h_{1}$ and the fact that $\Psi_{1} \Psi_{2}$ is decreasing function.

Next, we show that the sum

$$
\sum_{h=1}^{\infty} h^{n-2} \Psi_{3}(h)
$$

diverges. Assume that the sum (24) converges. Then, by the monotonicity of $\Psi_{3}$, we have

$$
l^{n-1} \Psi_{3}(l) \ll \sum_{l / 2 \leq h<l} h^{n-2} \Psi_{3}(h) \rightarrow 0 \quad \text { as } \quad l \rightarrow \infty .
$$

It follows that $l^{n-1} \Psi_{3}(l)=\min \left\{l^{n-1} \Psi_{1}(l) \Psi_{2}(l) ; 1\right\} \rightarrow 0$ as $l \rightarrow \infty$. This is possible only if $l^{n-1} \Psi_{1}(l) \Psi_{2}(l) \rightarrow 0$ as $l \rightarrow \infty$. It follows that $\Psi_{3}(l)=\Psi_{1}(l) \Psi_{2}(l)$ for all sufficiently large $l$. Therefore, the sum $\sum_{h=1}^{\infty} h^{n-2} \Psi_{1}(h) \Psi_{2}(h)$ converges, contrary to the fact that this sum diverges.

Using the monotonicity of $\Psi_{3}$ we obtain the following inequalities:

$$
2^{(t+1)(n-1)} \Psi_{3}\left(2^{t+1}\right) \ll \sum_{2^{t} \leq h<2^{t+1}} h^{n-2} \Psi_{3}(h) \ll 2^{t(n-1)} \Psi_{3}\left(2^{t}\right) .
$$

Summing these over all $t \in \mathbb{N}$ gives that the sums

$$
\sum_{h=1}^{\infty} h^{n-2} \Psi_{3}(h) \quad \text { and } \quad \sum_{t=0}^{\infty} 2^{t(n-1)} \Psi_{3}\left(2^{t}\right)
$$

(converge or) diverge simultaneously. 
The ubiquitous system. Define the functions $\overline{\bar{\Psi}}_{1}(q):=\frac{\bar{\Psi}_{1}(q)}{K^{2}}$ and $\bar{\Psi}_{2}(q):=\frac{\Psi_{2}(q)}{K}$, where $q^{\frac{-n+2}{3}} \leq \bar{\Psi}_{2}(q)<k_{0} q$ and $K=\left(3 v^{-1}\right)^{n-1} c_{1} n$.

Let $n \geq 2$. Let $\mathcal{R}$ be the set of algebraic numbers $\alpha_{1} \in \mathbb{R}$ of degree $n$ such that

$$
\left|\alpha_{1}-\alpha_{2}\right| \leq v^{-1} H\left(\alpha_{1}\right)^{-1} \bar{\Psi}_{2}\left(H\left(\alpha_{1}\right)\right) \text { for some } \alpha_{2} \in \mathbb{R} \text {, conjugate to } \alpha_{1}
$$

and

$$
\left|\alpha_{i}\right| \ll v^{-1} \text { for any } \alpha_{i} \in \mathbb{C} \text {, conjugate to } \alpha_{1} \text {, }
$$

where the constant implied by the Vinogradov symbol depends on $n$ only. We will identify $\mathcal{J}$ with $\mathcal{R}$, so that formally $r_{\alpha}=\alpha$. Furthermore, let $\beta_{\alpha}=\nu H(\alpha)$ and $\rho(q)=q^{-n+1} / \bar{\Psi}_{2}^{2}(q)$. Then, by Lemma 2 and Remark 1, there is a constant $v$ such that $(\mathcal{R}, \beta)$ is locally ubiquitous in $I:=\left[-\frac{1}{2}, \frac{1}{2}\right]$ with respect to the above $\rho$. Let $\psi(q)=\overline{\bar{\Psi}}_{1}(q) / \bar{\Psi}_{2}(q)$. If $\psi$ or $\rho$ is 2-regular function then Lemma 3 is applicable. Then $\frac{\psi\left(2^{t}\right)}{\rho\left(2^{t}\right)}=\overline{\bar{\Psi}}_{1}\left(2^{t}\right) \bar{\Psi}_{2}\left(2^{t}\right) 2^{t(n-1)}=K^{-3} \Psi_{3}\left(2^{t}\right) 2^{t(n-1)}$ and, therefore by (25), the sum $\sum_{t=1}^{\infty} \frac{\psi\left(2^{t}\right)}{\rho\left(2^{t}\right)}$ diverges.

Furthermore, we show that

$$
\Lambda_{\mathcal{R}}(\psi) \subset \mathcal{P}_{n}\left(c_{0} \overline{\bar{\Psi}}_{1}, c_{0} n \bar{\Psi}_{2}\right) \subset \mathcal{P}_{n}\left(\bar{\Psi}_{1}, \Psi_{2}\right) \subseteq \mathcal{P}_{n}\left(\Psi_{1}, \Psi_{2}\right)
$$

First, we show that $\Lambda_{\mathcal{R}}(\psi) \subset \mathcal{P}_{n}\left(c_{0} \overline{\bar{\Psi}}_{1}, c_{0} n \bar{\Psi}_{2}\right)$. By definition, for every $x \in \Lambda_{\mathcal{R}}(\psi)$ there are infinitely many real algebraic numbers $\alpha_{1}$ of degree $n$ satisfying (26), (27) and

$$
\left|x-\alpha_{1}\right|<\overline{\bar{\Psi}}_{1}\left(v H\left(\alpha_{1}\right)\right) / \bar{\Psi}_{2}\left(v H\left(\alpha_{1}\right)\right) .
$$

Let $\psi$ be a quasi-monotonic function, then we have that $\psi(v q) \leq c_{1} \psi(q)$ for some $c_{1} \geq 1$ and $v, 0<v<1$.

Let $P$ denote the minimal polynomial of $\alpha_{1}$. Then, $P(x)=a_{n}\left(x-\alpha_{1}\right) \ldots\left(x-\alpha_{n}\right)$. By (26), (27) and (29) we get

$$
\begin{aligned}
\left|x-\alpha_{1}\right| & <c_{1} \overline{\bar{\Psi}}_{1}(H) / \bar{\Psi}_{2}(H), \\
\left|x-\alpha_{2}\right| & \leq\left|x-\alpha_{1}\right|+\left|\alpha_{1}-\alpha_{2}\right|<c_{1} \overline{\bar{\Psi}}_{1}(H) / \bar{\Psi}_{2}(H)+v^{-1} H^{-1} \bar{\Psi}_{2}(H), \\
\left|x-\alpha_{k}\right| & \leq\left|x-\alpha_{1}\right|+\left|\alpha_{1}-\alpha_{2}\right|+\left|\alpha_{2}\right|+\left|\alpha_{k}\right|<c_{1} \overline{\bar{\Psi}}_{1}(H) / \bar{\Psi}_{2}(H) \\
& +v^{-1} H^{-1} \bar{\Psi}_{2}(H)+2 v^{-1} \text { for } 3 \leq k \leq n .
\end{aligned}
$$

The two estimates $\bar{\Psi}_{2}^{2}(H) \geq K^{3} H \overline{\bar{\Psi}}_{1}(H)$ and $\bar{\Psi}_{2}(H)<k_{0} H$ together with the fact that $\left|a_{n}\right| \leq H(P)$ give

$$
|P(x)|<H c_{1} \overline{\bar{\Psi}}_{1}(H) \bar{\Psi}_{2}^{-1}(H) 2 v^{-1} H^{-1} \bar{\Psi}_{2}(H)\left(3 v^{-1}\right)^{n-2}=c_{0} \overline{\bar{\Psi}}_{1}(H)
$$

with $c_{0}=2 \cdot 3^{n-2} c_{1} v^{1-n}$.

Furthermore,

$$
P^{\prime}(x)=a_{n} \sum_{i=1}^{n} \frac{\left(x-\alpha_{1}\right) \cdots\left(x-\alpha_{n}\right)}{\left(x-\alpha_{i}\right)} .
$$


Estimate every summand in (31) using (30):

$$
\begin{aligned}
& \left|x-\alpha_{2}\right| \cdots\left|x-\alpha_{n}\right|<2 \cdot 3^{n-2} v^{1-n} H^{-1} \bar{\Psi}_{2}(H), \\
& \left|x-\alpha_{1}\right|\left|x-\alpha_{3}\right| \cdots\left|x-\alpha_{n}\right|<c_{1} \overline{\bar{\Psi}}_{1}(H) / \bar{\Psi}_{2}(H)\left(3 v^{-1}\right)^{n-2}, \\
& \frac{\left|x-\alpha_{1}\right| \cdots\left|x-\alpha_{n}\right|}{\left|x-\alpha_{i}\right|}<2 \cdot 3^{n-3} v^{2-n} c_{1} \overline{\bar{\Psi}}_{1}(H) H^{-1} \quad \text { for } \quad 3 \leq i \leq n .
\end{aligned}
$$

Again, by the previous formulae, the estimates $\bar{\Psi}_{2}^{2}(H) \geq K^{3} H \overline{\bar{\Psi}}_{1}(H), \overline{\bar{\Psi}}_{1}(H) \leq \bar{\Psi}_{2}(H)$, $\bar{\Psi}_{2}(H)<k_{0} H$, and the fact that $\left|a_{n}\right| \leq H(P)$, we get $\left|P^{\prime}(x)\right|<c_{0} n \bar{\Psi}_{2}(H)$.

Second, we show that $\mathcal{P}_{n}\left(c_{0} \overline{\bar{\Psi}}_{1}, c_{0} n \bar{\Psi}_{2}\right) \subset \mathcal{P}_{n}\left(\bar{\Psi}_{1}, \Psi_{2}\right)$. Using the fact that $\bar{\Psi}_{2}(q)=$ $K^{-1} \Psi_{2}(q)$, we obtain

$$
\left|P^{\prime}(x)\right|<c_{0} n \bar{\Psi}_{2}(H)=c_{0} n K^{-1} \Psi_{2}(H)<\Psi_{2}(H) \quad \text { for } \quad K>c_{0} n .
$$

Similarly, we obtain that

$$
|P(x)|<\bar{\Psi}_{1}(H) \text { for } K>c_{0}^{1 / 2} .
$$

Third, by definition we have $\mathcal{P}_{n}\left(\bar{\Psi}_{1}, \Psi_{2}\right) \subseteq \mathcal{P}_{n}\left(\Psi_{1}, \Psi_{2}\right)$. Thus (28) is established and the proof of Theorem 1 in the divergence case is completed.

3. Proof of Theorem 1: the convergence case. First of all, note that since the sum $\sum_{H=1}^{\infty} H^{n-2} \Psi_{1}(H) \Psi_{2}(H)$ converges and $\Psi_{1} \Psi_{2}$ is monotonically decreasing, we have

$$
2^{-n+1} h^{n-1} \Psi_{1}(h) \Psi_{2}(h) \leq \sum_{h / 2 \leq l \leq h} l^{n-2} \Psi_{1}(l) \Psi_{2}(l) \rightarrow 0 \quad \text { as } \quad h \rightarrow \infty .
$$

This implies the inequality

$$
\Psi_{1}(h) \Psi_{2}(h) \leq h^{-n+1},
$$

valid for sufficiently large $h$, which will be used later on.

Let $\Psi_{2}(H) \geq H^{1 / 2+\epsilon}, \epsilon>0$. Furthermore, we will distinguish two cases for the value of the first derivative: $\left|P^{\prime}(x)\right|<H^{1 / 2}$ and $H^{1 / 2} \leq\left|P^{\prime}(x)\right|<\Psi_{2}(H)$.

3.1. Case I: $\left|P^{\prime}(x)\right|<H^{1 / 2}$. In this case, we use the result of Bernik et al. [18]. Using the notation in Theorem $1.4[\mathbf{1 8}]$, we take $\mathbf{f}=\left(x, x^{2}, \ldots, x^{n}\right), d=1, U=\mathbb{R}$ and $T_{1}=\cdots=T_{n}=H$, to obtain the following result.

Lemma 4. Let $x_{0} \in \mathbb{R}$ and $\delta_{0}=\max \left(\delta,\left(\delta K_{\infty} H^{n-1}\right)^{\frac{1}{n+1}}\right)$. Then there exists a finite interval $I_{0} \subset \mathbb{R}$ containing $x_{0}$ and a constant $E>0$ such that the set

$$
\cup_{P \in P_{n}, 0<H(P) \leq H}\left\{x \in I_{0}:|P(x)|<\delta,\left|P^{\prime}(x)\right|<K_{\infty}\right\}
$$

has measure at most $E \delta_{0}^{\frac{1}{2 n-1}}$.

Since $\Psi_{2}(H) \geq H^{1 / 2+\epsilon}$ and $\Psi_{1}(H) \leq \Psi_{2}(H)^{-1} H^{-n+1}$, then we get

$$
|P(x)|<\Psi_{1}(H) \leq H^{-n+1 / 2-\epsilon} .
$$


For a non-negative integer $r$, we denote by $\mathcal{A}(r)$ the set of $x \in I_{0}$ such that the system of inequalities

$$
|P(x)|<H(P)^{-n+1 / 2-\epsilon},\left|P^{\prime}(x)\right|<H(P)^{1 / 2},
$$

holds for some $P \in P_{n}$ with $2^{r-1} \leq H(P)<2^{r}$. According to Lemma $4, \mu(\mathcal{A}(r)) \ll$ $2^{\frac{-\epsilon r}{(n+1)(2 n-1)}}$ with $\epsilon>0$. The set of $x \in \mathbb{R}$ for which there are infinitely many $P \in P_{n}$ satisfying (33) consists of points $x \in \mathbb{R}$ which belong to infinitely many sets $\mathcal{A}(r)$. The sum $\sum_{r=1}^{\infty} \mu(\mathcal{A}(r))$ converges and the Borel-Cantelli lemma can be used to complete the proof in this case.

3.2. Case II: $H^{1 / 2} \leq\left|P^{\prime}(x)\right|<\Psi_{2}(H)$. Let $H>0$ be a sufficiently large number. Let $P_{n}(H)$ denote the set of polynomials $P \in P_{n}$ such that $H(P)=H$. Obviously $P_{n}=\cup_{H=1}^{\infty} P_{n}(H)$.

Given a polynomial $P \in P_{n}(H)$ with roots $\alpha_{1}, \alpha_{2}, \ldots, \alpha_{n} \in \mathbb{C}$, define the sets

$$
S\left(\alpha_{j}\right)=\left\{x \in \mathbb{R}:\left|x-\alpha_{j}\right|=\min _{1 \leq m \leq n}\left|x-\alpha_{m}\right|\right\}, 1 \leq j \leq n .
$$

When investigating the measure of $x \in \mathcal{P}_{n}\left(\Psi_{1}, \Psi_{2}\right)$ there is no loss of generality in restricting $x$ to belong to $S\left(\alpha_{j}\right)$ for a fixed $j$ for $P$ satisfying the inequalities within (1). For simplicity, throughout the proof we will assume that $j=1$.

We will use the following auxiliary statements which are established in [14, Lemma 2] and [25, p. 13].

Lemma 5. If $P \in P_{n}(H)$ and $x \in S\left(\alpha_{1}\right)$ then

$$
\left|x-\alpha_{1}\right| \leq n|P(x)|\left|P^{\prime}(x)\right|^{-1} \quad \text { for } \quad\left|P^{\prime}(x)\right| \neq 0,
$$

and

$$
\left|x-\alpha_{1}\right|<2^{n} \min \left(|P(x)|\left|P^{\prime}\left(\alpha_{1}\right)\right|^{-1},|P(x)|\left|P^{\prime}\left(\alpha_{1}\right)\right|^{-1}\left|\alpha_{1}-\alpha_{2}\right|^{1 / 2}\right) \text { for }\left|P^{\prime}\left(\alpha_{1}\right)\right| \neq 0 .
$$

Let $x \in\left[-\frac{1}{2}, \frac{1}{2}\right] \cap S\left(\alpha_{1}\right)$. Then from Lemma 5 and from the estimations for $\Psi_{1}$ and $\left|P^{\prime}(x)\right|$, we obtain

$$
\left|x-\alpha_{1}\right| \leq n|P(x)|\left|P^{\prime}(x)\right|^{-1}<n \Psi_{1}(H) H^{-1 / 2} \leq n \Psi_{2}(H)^{-1} H^{-n+1 / 2} .
$$

At the beginning, we obtain that the first derivative in the closest root to $x$ has the same order as $\left|P^{\prime}(x)\right|$. Using the mean value theorem, we obtain

$$
P^{\prime}(x)=P^{\prime}\left(\alpha_{1}\right)+P^{\prime \prime}\left(\beta_{1}\right)\left(x-\alpha_{1}\right), \quad \beta_{1} \in\left(x, \alpha_{1}\right) .
$$

Since $\left|P^{\prime \prime}\left(\beta_{1}\right)\left(x-\alpha_{1}\right)\right|<2^{4} n \Psi_{2}(H)^{-1} H^{3 / 2-n}$ and $\Psi_{2}(H) \geq H^{1 / 2+\epsilon}$ then from previous equality for sufficiently large $H$ we get

$$
\frac{1}{2} H^{1 / 2}<\left|P^{\prime}\left(\alpha_{1}\right)\right|<2 \Psi_{2}(H) .
$$


Now, for a given polynomial $P \in P_{n}(H)$, let

$$
\begin{aligned}
& \mathcal{L}_{1}(P)=\left\{x \in\left[-\frac{1}{2}, \frac{1}{2}\right] \cap S\left(\alpha_{1}\right):|P(x)|<\Psi_{1}(H), H^{1 / 2} \leq\left|P^{\prime}(x)\right|<\Psi_{2}(H)\right\}, \\
& \mathcal{L}_{2}(P)=\left\{x \in\left[-\frac{1}{2}, \frac{1}{2}\right] \cap S\left(\alpha_{1}\right):|P(x)|<\Psi_{1}(H), \frac{1}{2} H^{1 / 2} \leq\left|P^{\prime}\left(\alpha_{1}\right)\right|<2 \Psi_{2}(H)\right\} .
\end{aligned}
$$

Next, we will show that $\mu\left(\cap_{N=1}^{\infty} \cup_{H \geq N} \cup_{P \in P_{n}(H)} \mathcal{L}_{2}(P)\right)=0$. From Lemma 5 and the fact that $|P(x)|<\Psi_{1}(H)$, we obtain

$$
\left|x-\alpha_{1}\right|<2^{n} \Psi_{1}(H)\left|P^{\prime}\left(\alpha_{1}\right)\right|^{-1} .
$$

Let $\sigma(P)$ denote the set of solutions of the inequality (37) for a fixed $P \in P_{n}(H)$. Clearly, $\mathcal{L}_{1}(P) \subseteq \mathcal{L}_{2}(P) \subseteq \sigma(P)$

For $P \in P_{n}(H)$, consider the interval $\sigma_{1}(P)$ defined by the inequality

$$
\sigma_{1}(P):\left|x-\alpha_{1}\right|<\frac{1}{16 n}\left|P^{\prime}\left(\alpha_{1}\right)\right|^{-1} .
$$

There is a sufficiently large $H_{0}$ depending on $n$ such that $\sigma(P) \subset \sigma_{1}(P)$ for all $H>H_{0}$.

First, we consider the polynomials $P \in P_{n}(H)$ such that $a_{j}=H$ for some $j \geq 2$. The coefficient $a_{1}$ of the polynomial $P(x)=a_{n} x^{n}+a_{n-1} x^{n-1}+\cdots+a_{1} x+a_{0} \in P_{n}(H)$ can be written in the following form $a_{1}=\left[10 \Psi_{2}(H)\right] k+t$, where $0 \leq t \leq\left[10 \Psi_{2}(H)\right]-1$ and $|k|<\left[H\left(10 \Psi_{2}(H)-1\right)^{-1}\right]$.

Two polynomials

$$
\begin{aligned}
& P_{1}(x)=a_{1, n} x^{n}+a_{1, n-1} x^{n-1}+\cdots+H x^{j}+\cdots+a_{1,2} x^{2}+\left(\left[10 \Psi_{2}(H)\right] k_{1}+t_{1}\right) x+a_{1,0}, \\
& P_{2}(x)=a_{2, n} x^{n}+a_{2, n-1} x^{n-1}+\cdots+H x^{j}+\cdots+a_{2,2} x^{2}+\left(\left[10 \Psi_{2}(H)\right] k_{2}+t_{2}\right) x+a_{2,0}
\end{aligned}
$$

are included in the same class $P_{b_{t}}(H)$ if they have the same vector $b_{t}=$ $\left(a_{1, n}, a_{1, n-1}, \ldots, H, \ldots, a_{1,2}, t_{1}\right)$, where

$$
a_{1, n}=a_{2, n}, a_{1, n-1}=a_{2, n-1}, \ldots, a_{1, j}=a_{2, j}=H, \ldots, a_{1,2}=a_{2,2}, t_{1}=t_{2} .
$$

The number of different classes $P_{b_{t}}(H)$ is $\ll H^{n-2} \Psi_{2}(H)$.

Fix the vector $b_{t}=\left(a_{n}, a_{n-1}, \ldots, H, \ldots, a_{2}, t\right)$. Develop the polynomials $P(x) \in$ $P_{b_{t}}(H)$ as Taylor series in $\sigma_{1}(P)$ to obtain an upper bound for $|P(x)|$ so that

$$
P(x)=\sum_{j=1}^{n}(j !)^{-1} P^{(j)}\left(\alpha_{1}\right)\left(x-\alpha_{1}\right)^{j} .
$$

Estimating each term of the Taylor series individually gives

$$
\begin{aligned}
& \left|P^{\prime}\left(\alpha_{1}\right)\right|\left|x-\alpha_{1}\right|<\frac{1}{16 n}, \\
& 1 / j !\left|P^{(j)}\left(\alpha_{1}\right)\right|\left|x-\alpha_{1}\right|^{j}<\frac{1}{8 n^{2}}, 2 \leq j \leq n .
\end{aligned}
$$

Clearly, these further imply that

$$
|P(x)|<\frac{1}{5 n} .
$$


Furthermore, from the mean value theorem and from the estimations (36), (38) and $\Psi_{2}(H) \geq H^{1 / 2+\epsilon}$, for $x \in \sigma_{1}(P)$, we obtain

$$
\left|P^{\prime}(x)\right| \leq\left|P^{\prime}\left(\alpha_{1}\right)\right|+\left|P^{\prime \prime}\left(\beta_{2}\right)\right|\left|x-\alpha_{1}\right|<2 \Psi_{2}(H)+\frac{2}{n} H^{1 / 2}<3 \Psi_{2}(H),
$$

$\beta_{2} \in\left(x, \alpha_{1}\right)$.

It will now be shown that if $P, Q \in P_{b_{t}}(H)$ and $P \neq Q$ then the intervals $\sigma_{1}(P)$ and $\sigma_{1}(Q)$ are disjoint. Assume that this is not the case so that $\sigma_{1}(P) \cap \sigma_{1}(Q) \neq 0$. Let $R(x)=P(x)-Q(x) \in \mathbb{Z}_{\neq 0}$, then $R$ is of the form $R(x)=b_{1} x+b_{0}$ with $\left|b_{1}\right|=\mid a_{1}(P)-$ $a_{1}(Q)\left|=\left[10 \Psi_{2}(H)\right]\right| k(P)-k(Q) \mid$. It can be readily verified from (39) and (40) that

$$
\begin{aligned}
& \left|b_{1} x+b_{0}\right|<\frac{2}{5 n}, \\
& \left|b_{1}\right|<6 \Psi_{2}(H) .
\end{aligned}
$$

Since $\left|b_{1}\right|=\left[10 \Psi_{2}(H)\right]\left|k_{1}\right| \geq\left[10 \Psi_{2}(H)\right]$ for $k_{1} \in \mathbb{Z} /\{0\}$, therefore we have a contradiction with (41). Hence, there is no such $x$ and $\sigma_{1}(P) \cap \sigma_{1}(Q)=0$. Therefore,

$$
\sum_{P \in P_{b_{t}}(H)} \mu\left(\sigma_{1}(P)\right) \ll 1 .
$$

Also, from (37) and (38),

$$
\mu(\sigma(P)) \ll \mu\left(\sigma_{1}(P)\right) \Psi_{1}(H) .
$$

Since the number of classes $P_{b_{t}}(H)$ is at most $c(n) H^{n-2} \Psi_{2}(H)$ from the above two displayed inequalities, we have

$$
\sum_{b_{t}} \sum_{P \in P_{b_{t}}(H)} \mu(\sigma(P)) \ll H^{n-2} \Psi_{1}(H) \Psi_{2}(H) .
$$

By the condition of the theorem the series $\sum_{H=1}^{\infty} H^{n-2} \Psi_{1}(H) \Psi_{2}(H)$ converges and the Borel-Cantelli lemma shows that the set of $x$ belonging to infinitely many sets of $\sigma(P)$ has measure zero.

Second, we consider the polynomials $P \in P_{n}(H)$ such that $a_{0}=H$ or $a_{1}=H$. Fix $\theta>0$. As $\theta$ is arbitrary we may assume without loss of generality that any real number $x$ lying in the interval $\left[-\frac{1}{2}, \frac{1}{2}\right]$ satisfies $|x| \geq \theta$. For $n=2$, the proof is similar to that used by Sprindžuk in [25]. For $n \geq 3$, we pass from the polynomial $P$ to the reciprocal polynomial $Q(x)=x^{n} P\left(\frac{1}{x}\right)$ of $P$. Under such a transformation and its inverse, the measure of solutions $x$ changes in $c(n, \theta)$ times. Using $H(P)=H(Q), \Psi_{2}(H) \geq H^{1 / 2+\epsilon}$, $\theta \leq|x| \leq 1 / 2$ and (32), we verify that $|Q(x)| \ll \Psi_{1}(H)$ and $\left|Q^{\prime}(x)\right| \ll \Psi_{2}(H)$. Then for the polynomials $Q$ we use the same argument as before for $P$. The proof of Theorem 1 in the convergence case is completed.

ACKNOWLEDGEMEnTs. The author is grateful to Detta Dickinson and Vasili Bernik for their constant support and encouragement and to Victor Beresnevich for his helpful comments on an earlier draft of this paper.

\section{REFERENCES}

1. D. Badziahin, V. Beresnevich and S. Velani, Inhomogeneous theory of dual Diophantine approximation on manifolds, Preprint, http://arxiv.org/abs/1009.5638 (2010), pp. 1-37. 

1975).

2. A. Baker, Transcendental number theory (Cambridge University Press, Cambridge, UK,

3. V. Beresnevich, On approximation of real numbers by real algebraic numbers, Acta Arith. 90 (1999), 97-112.

4. V. Beresnevich, A Groshev type theorem for convergence on manifolds, Acta Math. Hungar. 94 (2002), 99-130.

5. V. Beresnevich, On a theorem of V. Bernik in the metric theory of Diophantine approximation, Acta Arith. 117(1) (2005), 71-80.

6. V. Beresnevich, Rational points near manifolds and metric Diophantine approximation, Preprint, http://arxiv.org/abs/0904.0474 (2009), pp. 1-46.

7. V. Beresnevich, V. I. Bernik and M. M. Dodson, Regular systems, ubiquity and Diophantine approximation, in A panorama of number theory or the view from Baker's garden (Zürich, 1999) (G. Wüstholz, editor) (Cambridge University Press, Cambridge, UK, 2002), pp. 260-279.

8. V. Beresnevich, D. Dickinson and S. Velani, Measure theoretic laws for lim sup sets, Mem. Amer. Math. Soc. 179 (2006), x+91.

9. V. Beresnevich, D. Dickinson and S. Velani, Diophantine approximation on planar curves and the distribution of rational points, Ann. Math. 166(2) (2007), 367-426. With an Appendix II by R. C. Vaughan.

10. V. Beresnevich, V. Bernik and F. Götze, The distribution of close conjugate algebraic numbers, Compositio Math. 146 (2010), 1165-1179.

11. V. V. Beresnevich, V. I. Bernik, D. Y. Kleinbock and G. A. Margulis, Metric Diophantine approximation: the Khintchine-Groshev theorem for nondegenerate manifolds, Mosc. Math. J. 2 (2002), 203-225.

12. V. Beresnevich, V. I. Bernik and E. I. Kovalevskaya, On approximation of $p$-adic numbers by $p$-adic algebraic numbers, J. Number Theory 111(1) (2005), 33-56.

13. V. I. Bernik, On the exact order of approximation of zero by values of integral polynomials, Acta Arith. 53 (1989), 17-28 (in Russian).

14. V. Bernik, O. Kukso and F. Götze, Lower bounds for the number of integer polynomials with given order of discriminants, Acta Arithm. 133 (2008), 375-390.

15. V. Bernik, N. Budarina and D. Dickinson, A divergent Khintchine theorem in the real, complex, and $p$-adic fields, Lith. Math. J. 48(2) (2008), 158-173.

16. V. Bernik, N. Budarina and D. Dickinson, Khinchin's theorem and the approximation of zero by values of integer polynomials in different metrics, Dokl. Akad. Nauk 413(2) (2007), $151-153$.

17. V. Bernik, N. Budarina and D. Dickinson, Simultaneous Diophantine approximation in the real, complex and p-adic fields, Math. Proc. Cam. Phil. Soc. 149(2) (2010), 217-227.

18. V. I. Bernik, D. Kleinbock and G. A. Margulis, Khintchine-type theorems on manifolds: the convergence case for standard and multiplicative versions, Int. Math. Res. Notices 2001(9) (2001), 453-486.

19. V. Bernik and N. Shamukova, Approximation of real numbers by integer algebraic numbers, and the Khinchin theorem, Dokl. Nat. Akad. Nauk Belarusi. 50(3) (2006), 30-32.

20. V. I. Bernik and D. V. Vasil'ev, A Khinchin-type theorem for integer-valued polynomials of a complex variable, Proceedings of the Institute of Mathematics, Vol. 3 (Russian), pp. 10-20.

21. N. Budarina and D. Dickinson, Diophantine approximation on non-degenerate curves with non-monotonic error function, Bull. Lond. Math. Soc. 41(1) (2009), 137-146.

22. Y. Bugeaud, Approximation by algebraic integers and Hausdorff dimension, J. Lond. Math. Soc. 65 (2002), 547-559.

23. A. J. Khintchine, Einige Satze uber Kettenbruche, mit Anwendungen auf die Theorie der Diophantischen Approximationen, Math. Ann. 92 (1924), 115-125.

24. D. Y. Kleinbock and G. A. Margulis, Flows on homogeneous spaces and Diophantine approximation on manifolds, Ann. Math. 148(2) (1998), 339-360.

25. V. Sprindžuk, Mahler's problem in the metric theory of numbers, Translations of Mathematical Monographs, Vol. 25 (American Mathematical Society, Providence, RI, 1969).

26. R. C. Vaughan and S. Velani, Diophantine approximation on planar curves: the convergence theory, Invent. Math. 166 (2006), 103-124. 